\title{
Caloric Intake and its Effect on Aging and Cognitive Slowing: A Review of the Research into Intermittent Fasting
}

\author{
Lindsay Talemal, BS* \\ Lewis Katz School of Medicine, Temple University, USA
}

*Corresponding author: Lindsay Talemal, Lewis Katz School of Medicine, Temple University, Philadelphia, PA, USA

\begin{abstract}
Aging has been defined as the biological changes occurring during a lifetime that result in a decreased resistance to cellular stress, more vulnerability to disease, and increased likelihood of death. Some mechanisms of aging that will be discussed in this paper include instability of genomes, neuroendocrine secreted factors, production of reactive oxygen species, altered calcium levels, and inflammation. Aging rates are species-specific, which suggests a strong genetic influence. However, one major variable shown to affect the rate of aging in many different species is caloric intake. Intermittent fasting involves increasing the interval between meals, therefore, lowering the daily caloric intake by approximately $40 \%$. Although cells in the body require energy from food in order to survive and function, an excessive amount of calories can compromise cell function over time and lead to detrimental health effects. Because of this, dietary restriction including intermittent fasting, can increase resistance to disease and also lifespan. Intermittent fasting has also been shown to improve memory, learning, and neurogenesis in the brain. Many recent studies have shown that dietary restriction has profound effects on the brain, and protects neurons against degeneration of Alzheimer's, Parkinson's and Huntington's diseases. Dietary restriction stimulates the production of new neurons from stem cells and enhances synaptic plasticity, which allows the brain to resist the changes of aging. These influences of the caloric intake on brain function and vulnerability to disease revealed new cellular mechanisms connected to diet that affect our nervous systems and are now leading to preventative and therapeutic approaches for neurodegenerative disorders.
\end{abstract}

\section{Introduction}

Many studies have been conducted on a population of people in southern Japan, the Okinawans, who have the world's highest life expectancy and the highest percentage of centenarians. They live by the rule 'Hara hatchi bu;' eating only until they are 80 percent full [1]. This is thought of as the secret to their extraordinary health and longevity. An initial hypothesis, the 'thriftygene' hypothesis, poses the idea that our genome has adapted through evolution to profit from minimal caloric intake in order to cope with scarce food resources. Because humans evolved in these limited nutrient environments, we had to adapt. When food supplies are scarce, cells are faced with stress that may induce changes in gene expression and result in adaptive changes in cellular metabolism. This fluctuation between starvation and satiety may have served as pressure to adapt our physiology to function optimally in an environment where resources were unpredictable [1]. However, this all changed when humans invented agriculture, thereby securing reasonably consistent food sources. When food supplies are plentiful, as in most human populations, at least in industrialized countries, individuals consume unnecessary amounts of calories.

Although food has classically been perceived as providing energy, its ability to prevent and protect against diseases is beginning to be recognized. Understanding this basis of effects of food on cognition will help to determine the best ways to manipulate diet to increase the resistance of neuronal aging and promote mental fitness. Recent research has provided exciting evidence for the influence of diet on specific cellular mechanisms that maintain mental function; one of the most significant diets being intermittent fasting. This concept of caloric restriction being linked to how humankind has evolved is directly related to Intermittent Fasting. It provides a clear reasoning for

\footnotetext{
Citation: Talemal L (2021) Caloric Intake and its Effect on Aging and Cognitive Slowing: A Review of the Research into Intermittent Fasting. J Nutri Med Diet Care 7:052. doi.org/10.23937/25723278/1510052

Accepted: September 02, 2021: Published: September 04, 2021

Copyright: @ 2021 Talemal L. This is an open-access article distributed under the terms of the Creative Commons Attribution License, which permits unrestricted use, distribution, and reproduction in any medium, provided the original author and source are credited.
} 
why the intermittent shocks of hunger have surprising and beneficial effects on the brain. There are three different types of intermittent fasting diets. They include the 5:2 plan, eating normally five days a week and consuming fewer than 600 calories the other two days, periodic fasting, eating normally for one day and then fasting the next, and time restrictive fasting, where you only eat between noon and 8 pm every day [2]. Although these regimens vary slightly in their approached, they all involve a steady disruption in the average flow of calories.

Since it was discovered that calorie-restricted laboratory mice lived twice as long as those that were well fed [1,2], scientists have continually pursued further caloric restriction research in the hopes of discovering strategies for extending life and preventing disease. The number of people with age-related neurodegenerative disorders such as Alzheimer's, Parkinson's and Huntington's is increasing as population life expectancy is increasing. The impact of these disorders on society is highlighted by the fact that each year more money is required from the government to care for these patients. Alzheimer's, Huntington's and Parkinson's all share the commonality that the brain's cells accumulate toxic proteins with aging, which impede the normal molecular functioning. Over time these proteins accumulate, and neurons die, however, there is evidence that now suggests getting rid of them helps to restore neuronal survival and delay symptoms. Several research groups have found that intermittent fasting can help improve neural connections and protect them from the accumulation of amyloid plaques, a type of toxic protein that are prevalent in those with Alzheimer's disease (AD) [2]. The possibility that intermittent fasting can reduce the risk of neurodegenerative disorders is supported by epidemiological studies. When comparing average daily food intake to the incidence of Alzheimer's disease in different populations, there is a correlation that suggests low food intake is associated with decreased disease incidence [1]. Overall, the death and degeneration of neurons that occurs in each of these disorders has been linked to mechanisms including oxidative damage and gene dysregulation. Intermittent fasting has been shown to ameliorate the disorders through various targets including synaptic plasticity, cellular stress, gene expression regulation, and secretion of important neurochemical factors, and these findings will be discussed further in this paper.

\section{Neurogenesis and Synaptic Plasticity for Increased Learning and Memory}

Brain networks associated with feeding seem to be closely associated with those involved in cognitive processing. Caloric restriction has been associated with enhanced memory consolidation and facilitation of synaptic plasticity in several studies [3,4]. In completing intermittent fasting experiments with humans in a consistently aging population, considered individuals aged $50-80$ years, it was discovered that there was a highly significant, $20 \%$, improvement in the calorie restricted group's ability to recall words they had on a list [4]. When given this test, also called delayed recall, the fasted group was also noted to make fewer errors than the control group. Their memory improvement was correlated with reductions in blood insulin and markers of inflammation.

In mice it was also shown that reducing calorie intake by $40 \%$ decreases deficits in both motor and cognitive function that are associated with the process of aging [5]. Using maze experiments with mice, animals in the calorie restricted groups exhibited a significantly shorter escape latency than control groups in multiple studies. Similarly, life-long caloric restriction was seen to prevent age-related deficits in the performance of rats in maze learning and memory tasks [5]. These results suggest that intermittent fasting can increase learning and memory function. These same mice, maintained on a diet of $40 \%$ reduction in calories, did not exhibit the deficits in motor coordination and spatial learning seen in aging control mice [3]. This is believed to be due to changes in long-term potentiation of synaptic transmission which is correlated to both learning and memory. Aging rats exhibit a deficit in long-term potentiation in the hippocampus, and this is largely abolished in similarly aged rats that are kept on an intermittent fasting diet during their adult life.

Intermittent fasting significantly improved prefrontal-cortex-related cognitive functions such as mental flexibility and shifting set in experiments with model organisms as well as humans, and this is of importance because the prefrontal complex is responsible for the highest-order cognitive abilities including executive functioning [3]. Fasting animals have been shown to develop protective measures against damage from mechanisms that cause degeneration in the brain [4]. The shock of fasting leads to the brain creating new cells through the process of neurogenesis. As more neurons grow, the brain becomes increasingly resistant to the effects of protein plaques that underlie cases of Alzheimer's and to the damage inflicted by disorders such as Parkinson's. Intermittent fasting was also shown to improve age-related deficits in cognitive function in a mouse model of Alzheimer's disease [5]. It was reported to reduce the development of amyloid plaques in the hippocampus and cerebral cortex, which is a main characteristic of Alzheimer's pathology. Neurotrophic factors, specifically Brain Derived Neurotrophic Factor (BDNF) which will be discussed later in this paper, have also been shown to modulate synaptic plasticity in ways that facilitate learning and memory. BDNF is involved in NMDA receptor activation through an increase in calcium, leading to additional post-synaptic BDNF release that increases the cycling of pre-synaptic vesicles, and ultimately enhances longterm potentiation and synaptic plasticity. 


\section{Combatting Cellular Stress and Senescence}

The beneficial effects of calorie restriction, particularly those of intermittent fasting, appear to be the result of a cellular stress response stimulating the production of proteins that enhance neuronal plasticity and resistance to oxidative and metabolic disfunction [6-8]. These proteins include neurotrophic factors such as BDNF, stress protein chaperones such as heat-shock proteins, and mitochondrial uncoupling proteins $[9,10]$. In intermittent fasting it has been shown that there is increased sympathetic activity, which comes across as a stress response physiologically [11]. Periods without food provide small amounts of stress on our genome, called hormetic stress, which is stress that is actually beneficial to the body [6]. Similarly to when muscles are exercised and the stress of weight is placed on them, the neurons in the brain also benefit from being mildly stressed due to minimizing their food intake.

These beneficial effects of dietary restriction on brain function and life span, however, seem to depend upon intermittent fasting's ability to acutely reduce levels of oxidative stress. Oxidative stress damage in the brain is caused by senescent decline of brain function, but fasting improves the functional capacity and protects the brain by reducing oxidative damage to proteins and DNA $[6,8,9]$. Oxidative damage is indicative of an increase in protein carbonyls and loss of protein sulfhydryls, which was shown to be reversed in calorie restricted subjects $[7,10]$. It is also reflected by the glutathione redox state, which increases in the aging brain and is linked to age-associated losses of function and neurodegenerative diseases. Intermittent fasting, implemented in mice, increased the GSH/GSSG (glutathione/oxidized glutathione) ratio and redox potential in the same brain regions that typically exhibit age-related decreases $[8,11]$. This all suggests that the suppression of oxidative stress is one mechanism for how intermittent fasting protects neurons.

Multiple studies also suggest that this $40 \%$ reduction in caloric intake through intermittent fasting increases calcium retention in the mitochondria, compared to in natural aging when intracellular calcium levels are high $[7,12,13]$. Alzheimer's disease and other neurological disorders cause an excessive influx of calcium ions from the mitochondria into brain due to over-activation of glutamate receptors. This excitotoxicity can damage and kill neurons. Intermittent fasting, however, increases levels of the protein SIRT3, which removes an acetyl group in a process known as deacetylation, and allows the mitochondria to retain more calcium [13]. In the brain, this helps to avoid neuronal death associated with these neurodegenerative conditions, especially since calcium participates in the process of communication between neurons. Fasting has also been shown to up-regulate SIRT1 and down-regulate cytokines including p53 and p16 [9]. SIRT1 in known to play a critical role in regulation of normal cognitive function and synaptic plasticity and has been implicated in regulating processes underlying longevity and delayed cellular senescence [7]. Products of inflammation and high calorie intake, including p53 and p16, have been shown to hamper cognitive function [14], and so a down regulation of these in fasting state constitutes a great health benefit.

Although eating does provide energy for the body, carbohydrates, fats and proteins in food are metabolized to glucose which is then utilized for ATP production, and excessive energy production may cause cells to become damaged and thereby susceptible to disease. Higher fuel availability also leads to increased frequency of mitochondrial respiration. This consequentially increases production of reactive oxygen species from the electron transport chain, which contain unpaired electrons making them highly reactive and destructive physiologically, which is a major hallmark of aging and specifically neurodegeneration $[8,12]$. Ketones, released during fasting, however, inhibit this production of free radicals and also stimulate the activity of the innate anti-oxidant system to combat the molecules using antioxidant enzymes including glutathione peroxidase, glutathione reductase and superoxide dismutase in the mitochondria $[12,13]$. This gives enhanced capacity to manage oxidative stress.

\section{Major Beneficial Secreted Factors during Intermittent Fasting}

From recent research, it appears that a major contributor to the beneficial effects of fasting on neurons comes from the cellular stress response discussed previously, in which levels neurotrophic factors, a family of proteins that are responsible for the growth, survival, and maintenance of nerve cells over time, have increased secretion $[15,16]$. In general, the neurotrophic factors seem to protect neurons by inducing expression of genes encoding proteins that produce antioxidant enzymes and stabilize cellular calcium homeostasis $[17,18]$. Several research studies have shown that some of these factors are capable of regenerating damaged neurons, specifically Brain Derived Neurotrophic Factor (BDNF) $[2,15,16,19]$. When the brain is challenged by physical exertion, cognitive tasks, and even more importantly caloric restriction through intermittent fasting, the body produces elevated levels of BDNF, which in turn strengthens neural connections and increases the production of neurogenesis $[13,17]$. Its role in neuroplasticity allows the brain to continue to change and reorganize, therefore making the brain more resilient to stress and more adaptable, while stimulating new connections and synapses to boost memory and learning capabilities [2]. Intermittent fasting has been shown to boost BDNF by $50-400 \%$ [15], and since research has also revealed that this factor causes an increase in the number of mitochondria in 
neurons, specifically in the hippocampus [18], it makes sense why model organisms on fasting cycles perform better at learning and memory challenges. The opposite is true for those on high calorie diets, especially since decreased levels of BDNF have been associated with neuronal loss leading to diseases such as Parkinson's, Alzheimer's, multiple sclerosis, and Huntington's [17].

Other secreted factors during fasting that have been shown to have beneficial effects on the body include Leptin and Ghrelin. Leptin, is a protein increased in a reduced appetite, as just like BDNF, leptin also facilitates synaptic plasticity. Leptin promotes rapid changes in hippocampal dendritic formations that suggest that it exerts direct action on hippocampal plasticity and, therefore, also has specific direct effects on memory [19]. Ghrelin is an adipogenic hormone that is secreted by an empty stomach during fasting as well. It promotes rapid reorganization of synaptic terminals in the hypothalamus, and in the hippocampus it promotes synapse formation in dendritic spines and increases long term potentiation, which enhances spatial learning and memory formation [20].

It has also been determined that levels of the ketone body $\beta$-hydroxybutyrate increase on a periodic fasting regimen, which suggests a change in the cellular energy metabolism pathways [21]. Ketones have been shown to promote positive changes in the structure of synapses that are important for learning, memory, and overall brain health $[14,21,22]$. Even more importantly, $\beta$-hydroxybutyrate, which is considered the principal ketone, has been shown to be a super-fuel that produces ATP energy more efficiently than glucose [22]. Mice on an intermittent fasting diet exhibited a 2 -fold increase in the concentration of $\beta$-hydroxybutyrate, and it has also been shown in multiple studies to protect neurons in animal models of Alzheimer's and Parkinson's diseases [21]. Not only does $\beta$-hydroxybutyrate seem to protect neuronal cells from toxins associated with Alzheimer's and Parkinson's, but it also seems to improve antioxidant function, increase mitochondria concentration, and stimulate neurogenesis [14]. It appears that the central nervous system has a greater ability of recovery and antiaging than scientists previously thought. The general view had been that the brain stopped developing after the first few years of life, however, this new research suggests that the brain can continue to reorganize itself by forming new neural connections with an intermittent fasting diet.

\section{Gene Regulation and Expression with Low Caloric Intake}

Aging is known to result in a gene-expression profile indicative of inflammation, oxidative stress, and reduced neurotrophic support in the brain. Caloric restriction through fasting, which combats the aging process, selectively weakens the age-associated induction of genes encoding these responses [23,24]. There is considerable evidence, through longitudinal research of birth, death, health and genealogical records, that an individual's risk for neurodegenerative disease is increased if their paternal grandparents grew up in times of food abundance rather than times of food shortage [9]. This is because fasting and low caloric intake exerts neuroprotective effects through inducing the expression of proteins that promote cell survival. It was previously discussed that two major classes of these survival proteins are protein chaperones and neurotrophic factors, which, when increased as in fasting, can protect neurons against excitotoxic and oxidative insults. One of the largest classes of transcripts induced by intermittent fasting is composed of growth and neurotrophic factors, and specifically the genetic deletion of the BDNF gene has been shown to impair memory formation $[9,25]$. It is known that the BDNF system is particularly susceptible to epigenetic modifications that influence cognitive function and that BDNF mRNA increases approximately 3.5 -fold following intermittent fasting $[24,25]$. Analysis of the levels of mRNAs encoding thousands of proteins in the brain after being on calorie restricted diets, revealed numerous age-related changes in gene expression [25]. The genes in which expression was primarily affected by aging, were counteracted by intermittent fasting and included those involved in the oxidative stress response, innate immunity, and in metabolism of energy $[9,23]$. The silent information regulator 2 gene (SIRT2), a member of the sirtuin protein family, has emerged in the research as an important modulator of cellular homeostasis and genomic stability which seems to act by silencing the function of specific aging genes. People on fasting diets, who had increased expression of one of the polymorphisms increasing SIRT1, showed better preservation of cognitive function and memory on all measurements than the control groups $[26,27]$.

\section{Conclusion and Further Directions}

Collectively, recent data suggests that reductions in caloric intake may protect the brain against aging and should be considered as an approach for reducing the risk of neurodegenerative disorders. The data obtained from both human and animal studies suggests that lowering daily calorie intake by $40 \%$ of calories dramatically reduces the risk of age-related disorders of the nervous system including Alzheimer's disease and Parkinson's disease, and that skipping meals through intermittent fasting is beneficial. Incorporating this knowledge into the design of treatments could be vital to combating mental diseases and understanding the molecular basis of the effects of food on cognition will help us to determine how to best manipulate diet to promote mental fitness. There is a lot to learn about the effects of food intake, specifically quantity, on the nervous system and its functional capabilities and, so, progress in this important area of investigation would be reinforced by the use of other model organisms in which genes that mediate these effects might be rapidly 
and easily identified. There is a wide complexity in the aging CNS and a lack of suitable known biomarkers of aging, therefore, approaches that could provide an assessment of this would increase understanding of the aging process and provide new tools for intervention. Intermittent caloric restriction seems to have health benefits in humans, but further preclinical information is required for the design of therapeutic applications and to further clarify this. One possible therapeutic agent that should be looked into with immediacy is BDNF, which seems as though it has great potential to combat neurodegenerative and metabolic diseases. As someone who was very skeptical of fasting until researching it myself, now seeing the research I believe the public should be made more aware of the devastating consequences of overeating. The current data suggests that the nervous system is highly vulnerable to excessive calorie intake, and that fasting can counteract the effects, so I feel that this information should be more widespread. Overall, it seems safe to say that if people implemented a sort of Okinawan approach to food intake, employing intermittent fasting, there would be a great reduction in the incidence of Alzheimer's, Parkinson's and other neurodegenerative disorders that currently plague society.

\section{References}

1. Grant W, et al. (1997) Dietary links to Alzheimer's disease. Alz Dis Rev 2: 42-55.

2. Mattson M, Longo VD, Harvie M (2017) Impact of intermittent fasting on health and disease processes. Ageing Research Reviews 39: 46-58.

3. Fontán-Lozano Á, Sáez-Cassanelli JL, Inda MC, de los Santos-Arteaga M, Sierra-Domínguez SA, et al. (2007) Caloric restriction increases learning consolidation and facilitates synaptic plasticity through mechanisms dependent on NR2B subunits of the NMDA receptor. The Journal of Neuroscience 27: 10185-10195.

4. Solianik R, Sujeta A, Terentjevienè A, Skurvydas A (2016) Effect of $48 \mathrm{~h}$ fasting on autonomic function, brain activity, cognition, and mood in amateur weight lifters. Biomed Res Int 2016: 1503956.

5. Stewart J, Mitchell J, Kalant N (1989) The effects of lifelong food restriction on spatial memory in young and aged Fischer 344 rats measured in the eight-arm radial and the Morris water mazes. Neurobiol Aging 10: 669-675.

6. Dubey A, Forster MJ, Lal H, Sohal RS (1996) Effect of age and caloric intake on protein oxidation in different brain regions and on behavioral functions of the mouse. Arch Biochem Biophys 333: 189-197.

7. Guarente L, Kenyon C (2000) Genetic pathways that regulate ageing in model organisms. Nature 408: 255-262.

8. Halagappa VKM, Anson Z, Pearson M, Matsuoka Y, Cutler RG, et al. (2007) Intermittent fasting and caloric restriction ameliorate age-related behavioral deficits in the tripletransgenic mouse model of Alzheimer's disease. Neurobiol Dis 26: 212-220.

9. Lee C, Weindruch R, Prolla TA (2000) Gene-expression profile of the ageing brain in mice. Nature News 25: $294-$ 297.
10. Xu B-L, Wang R, Ma L-N, Dong W, Zhao Z-W, et al. (2015) Effects of caloric intake on learning and memory function in juvenile C57BL/6J mice. Biomed Res Int 17.

11. Rebrin I, Forster MJ, Sohala RS (2006) Effects of age and caloric intake on glutathione redox state in brain regions of C57BL/6 and DBA/2 Mice. Brain Research 1127: 10-18.

12. Guo Z, et al. (2000) Beneficial effects of dietary restriction on cerebral cortical synaptic terminals: Preservation of glucose transport and mitochondrial function after exposure to amyloid $\beta$-peptide and oxidative and metabolic insults. J. Neurochem 75: 314-320.

13. Witte AV, Fobker M, Gellner R, Knecht S, Flöel A (2009) Caloric restriction improves memory in elderly humans. Proceedings of the National Academy of Sciences 106: 1255-1260.

14. Anson RM, Guo Z, Cabo R, Iyun T, Rios M, et al. (2003) Intermittent fasting dissociates beneficial effects of dietary restriction on glucose metabolism and neuronal resistance to injury from calorie intake. Proc Natl Acad Sci USA 100: 6216-6220.

15. Gómez-Pinilla F (2008) Brain Foods: The Effects of Nutrients on Brain Function. Nature Reviews Neuroscience 9: $568-578$.

16. Walsh JJ, Edgett BA, Tschakovsky ME, Gurd BJ (2015) Fasting and exercise differentially regulate BDNF MRNA expression in human skeletal muscle. Appl Physiol Nutr Metab 40: 96-98.

17. Bathina S, Das UN (2015) Brain-derived neurotrophic factor and its clinical implications. Archives of Medical Science 6: 1164-1178.

18. Lee J, et al. (2000) Dietary restriction increases survival of newly-generated neural cells and induces BDNF expression in the dentate gyrus of rats. J Mol Neurosci 15: 99-108.

19. Harvey J (2007) Leptin regulation of neuronal excitability and cognitive function. Curr Opin Pharmacol 7: 643-647.

20. van der Lelya AJ, Tschöp M, Heiman ML, Ghigo E (2004) Biological, physiological, pathophysiological, and pharmacological aspects of ghrelin. Endocr Rev 25: 426-457.

21. Kashiwaya Y, Takeshima T, Mori N, Nakashima K, Clarke $K$, et al. (2000) D- $\beta$-Hydroxybutyrate Protects Neurons in Models of Alzheimer's and Parkinson's Disease. Proceedings of the National Academy of Sciences of the United States of America 97: 5440-5444.

22. Cahill J, Veech RL (2003) Ketoacids? Good Medicine? Trans Am Clin Climatol Assoc 114: 149-161.

23. Duan W, Lee J, Guo Z, Mattson MP (2001) Dietary restriction stimulates BDNF production in the brain and thereby protects neurons against excitotoxic injury. $\mathrm{J}$ Mol Neurosci 16: 1-12.

24. Ingram DK, Weindruch $R$, Spangler EL, Freeman JR, Walford RL (1987) Dietary restriction benefits learning and motor performance of aged mice. J Gerontol 42: 78-81.

25. Linnarsson S, Björklund A, Ernfors $P$ (1997) Learning deficit in BDNF mutant mice. Eur J Neurosci 9: 2581-2587.

26. Mattson MP (2005) Energy intake, meal frequency, and health: A neurobiological perspective. Annu Rev Nutr 25: 237-260.

27. Mattson MP, Duan W, Guo Z (2003) Meal size and frequency affect neuronal plasticity and vulnerability to disease: Cellular and molecular mechanisms. Journal of Neurochemistry 84: 417-431. 